Recepción: 15 / 04 / 2017

Aceptación: 01 / 05 / 2017

Ciencias Médicas

Publicación: 15 / 05 / 2017

Articulo Científico

\title{
Manejo inicial de apendicitis aguda
}

\section{Initial management of acute appendicitis}

\section{O manejo inicial de apendicite aguda}

\author{
Mónica A. Altamirano-Gómez \\ altgom@hotmail.com
}

Efrén J. Aráuz-Perez" earauzperez@hotmail.com

Aurora B. Tutiven-Ubilla ${ }^{\mathrm{II}}$ auroratuti@hotmail.com

Correspondencia: altgom@hotmail.com

Magister en Emergencias Médicas; Especialista en Cirugía General; Doctora en Medicina y Cirugía; Profesora Auxiliar de la Universidad de Guayaquil Cátedra de Cirugía, Ecuador.

II. Especialista en Urología, Doctor en Medicina y Cirugía; Profesor Auxiliar de la Universidad de Guayaquil Cátedra de Urología, Ecuador.

III. Especialista en Nefrología; Doctora en Medicina y Cirugía; Universidad Católica Santiago de Guayaquil. Ecuador. 


\section{Resumen}

La apendicitis aguda se presenta en un $10 \%$ de la población occidental, teniendo como incidencia entre los 20 -25 años, aunque se puede observar en cualquier edad. Objetivo: Establecer la incidencia de apendicitis aguda y proponer un protocolo para el manejo de esta patología para el área de Emergencia del HAGP, investigación de tipo descriptiva Diseño no experimental retrospectivo. Las conclusiones de este trabajo fueron que los/as pacientes acuden forma directa a solicitar atención sin cumplir con el sistema de referencia y contrarreferencia, 45, 69\% fueron mujeres y 54,31\% hombres. Los más afectados menores de 19 años y de 20 a 29 años. El 66\% buscó atención médica antes de las 24 horas de la presentación de síntomas o signos, el 13\% entre 25 a 48 horas y el $21 \%$ después de las 49 horas. El 16\% presentó co-morbilidad, el 11\% presentó diabetes en todos los grupos de edad. Los signos presentes en la valoración fueron Blumberg Mc Burney Murphy en forma única o combinada. Las alteraciones hematológicas más frecuentes fueron Leucocitosis $\geq 10000 \mathrm{~mm} 366,37 \%$; Glicemia $\geq 120 \mathrm{mgl} 44 \%$; Creatinina sérica $\geq 1.13,45$. Los antibióticos utilizados fueron Ceftriaxona 36,21\%, Amplicilina 20,69\% Amplicilina sulbactam 8,62 $\%$ y no recibieron medicación profiláctica 26,72\%. El $96 \%$ recibió anestesia general y el $4 \%$ raquídea. Las complicaciones intra-operatoria fueron contaminación contenido apendicular $93 \%$ y $3,45 \%$ contaminación por coprolito y hemorragia controlada, respectivamente. Complicaciones post operatorias $81 \%$ infección de la herida, $11 \%$ abscesos intra-abdominales y $8 \%$, hematoma de pared. El $75 \%$ de los pacientes fue operado entre 1 a 4 horas posteriores al ingreso y más de $12 \mathrm{~h}$ en 13\%.El diagnóstico definitivo fue $40 \%$ apendicitis aguda, 48\% AA con peritonitis generalizada, $11 \%$ AA con absceso peritoneal.

Palabras clave: Apendicitis aguda; complicaciones; emergencia; protocolo; valoración. 


\begin{abstract}
Acute appendicitis occurs in $10 \%$ of the Western population, with the incidence among $20-25$ years, though can be observed at any age. Objective: To establish the incidence of acute appendicitis and propose a protocol for the management of this condition for the area's Emergency HAGP, descriptive research design is not experimental - retrospective. The findings of this study were that / as patients go directly to seek care without complying with the reference and counter, 45, 69\% were female and 54.31\% male. Most affected children under 19 years and 20-29 years. 66\% sought medical attention within 24 hours of the presentation of symptoms or signs, $13 \%$ between $25-48$ hours and $21 \%$ after 49 hours. $16 \%$ had co-morbidities, $11 \%$ had diabetes in all age groups. The signs were present in the valuation Blumberg Mc Burney Murphy in single or combined form. The most common hematologic abnormalities were leukocytosis $\geq 10000 \mathrm{~mm} 366.37 \%$; Glucose $\geq 120$ mgl 44\%; Serum creatinine $\geq 1.13 .45$. Ceftriaxone antibiotics used were $36.21 \%, 20.69 \%$ ampicillin + sulbactam $8.62 \%$ and $26.72 \%$ did not receive prophylactic medication. $96 \%$ received general anesthesia and spinal 4\%. The intra-operative complications were appendiceal pollution content 93\% and $3.45 \%$ contamination coprolite and controlled hemorrhage respectively. Post operative complications $81 \%$ wound infection, intra-abdominal $11 \%$ and $8 \%$ abscess, haematoma wall. $75 \%$ of patients underwent surgery between 1-4 hours of admission and $12 \mathrm{~h}$ in $13 \%$. The definitive diagnostic was acute appendicitis 40\%, 48\% AA with generalized peritonitis, 11\% AA with peritoneal abscess.
\end{abstract}

Key words: Apendicitis aguda; complicaciones; emergencia; protocolo; valoración. 


\section{Resumo}

A apendicite aguda ocorre em 10\% da população ocidental, com a incidência entre 20-25 anos, embora possa ser observada em qualquer idade. Objetivo: Estabelecer a incidência de apendicite aguda e propor um protocolo para o manejo dessa condição para a HAGP de emergência da área, o desenho descritivo de pesquisa não é experimental - retrospectivo. Os achados deste estudo foram que / como os pacientes vão diretamente para procurar atendimento sem cumprir com a referência e contador, 45, 69\% eram do sexo feminino e 54,31\% do sexo masculino. A maioria das crianças afetadas menores de 19 anos e 20-29 anos. 66\% procuraram atendimento médico dentro de 24 horas da apresentação dos sintomas ou sinais, 13\% entre 25-48 horas e 21\% após 49 horas. 16\% tinham co-morbidades, $11 \%$ tinham diabetes em todos os grupos etários. Os sinais estavam presentes na avaliação Blumberg Mc Burney Murphy em forma única ou combinada. As anomalias hematológicas mais comuns foram leucocitose $\geq 10000 \mathrm{~mm} 366,37 \%$; Glicose $\geq 120 \mathrm{mgl} 44 \%$; Creatinina sérica $\geq 1,13,45$. Os antibióticos ceftriaxona utilizados foram 36,21\%, 20,69\% ampicilina + sulbactam $8,62 \%$ e 26,72\% não receberam medicação profilática. 96\% receberam anestesia geral e 4\% espinhal. As complicações intra-operatórias foram conteúdo de poluição apendicular de $93 \%$ e 3,45\% de contaminação por coprolito e hemorragia controlada respectivamente. Complicações pósoperatórias $81 \%$ infecção da ferida, abcesso intra-abdominal 11\% e 8\%, parede do hematoma. O diagnóstico definitivo foi apendicite aguda 40\%, 48\% AA com peritonite generalizada, 11\% AA com abscesso peritoneal, sendo que $75 \%$ dos pacientes foram operados entre 1 a 4 horas de internação e $12 \mathrm{~h}$ em $13 \%$.

Palavras chave: Apendicite aguda; complicações; emergência; protocolo; avaliação. 


\section{Introducción.}

La apendicitis aguda es la principal causa de cirugía no electiva practicada en el mundo (Abdomen Quirúrgico Agudo) $^{1}$ se caracteriza por ser una reacción inflamatoria localizada en el apéndice cecal, ocasionada en la mayoría de la veces por una obstrucción de su luz, sin embrago la etiología en ocasiones no puede ser establecida con precisión. La apendicitis aguda se presenta en un $10 \%$ de la población occidental, teniendo como incidencia entre los 20 -25 años35aunque se puede observar en cualquier edad. Por razones desconocidas la mayor incidencia es en hombres con una relación de 1.4/1 con relación a las mujeres.

La primera Apendicectomía fue realizad por Claudius Armyad, cirujano de los hospitales de Westminister en 1736, pero en 1755 fue Heister que comprendió que el apéndice podía ser el asiento de la inflamación aguda primaria.29 Desde que Mc Burney introdujo en 1894 la apendicectomía como tratamiento de elección de la apendicitos aguda, se convirtió rápidamente en un procedimiento quirúrgico realizado con mucha frecuencia. La técnica permaneció sin modificaciones por más de un siglo ya que se combinaba la eficacia terapéutica y las bajas tasas de morbilidad y mortalidad. Con el desarrollo de la cirugía endoscópica se inició la realización de la apendicectomía laparoscópica descrita por Semm en 1983, sin embrago el nuevo método ha ganado una aceptación parcial, porque las ventajas no eran tan evidentes como las de la colecistectomía laparoscópica².

Es necesario reconocer sus primeros síntomas de la a apendicitis aguda, el diagnóstico puede ser difícil en niños, ancianos y mujeres en edad fértil y en nuestro medio se ha convertido en una de las causas más comunes de la cirugía abdominal de emergencia, 
En los últimos años la apendicitis aguda ha ido disminuyendo en frecuencia por razones poco claras, se aduce al avance de las ayudas diagnósticas y la conducta conservadora de los médicos, en años atrás los médicos eran más agresivos ante la sospecha de apendicectomía.

El procedimiento consiste en la extirpación quirúrgica del apéndice, un tubo de aproximadamente seis pulgadas de largo que se ramifica a partir del intestino grueso.

En la investigación Apendicectomía versus tratamiento con antibióticos para la apendicitis aguda, realizada por WilmsI, et al, los autores concluyeron que el límite superior del IC del 95\% del TAB para la curación en el transcurso de dos semanas sin complicaciones graves cruza el margen del $20 \%$ de la apendicectomía, por lo que el resultado no es concluyente. Además, la calidad de los estudios fue deficiente a moderada, por lo que los resultados se deben interpretar con precaución y no es posible establecer conclusiones definitivas. Por lo tanto, la apendicectomía es el tratamiento estándar para la apendicitis aguda. El tratamiento con antibióticos se puede utilizar como tratamiento alternativo en un ECA de buena calidad o en pacientes específicos o con afecciones en las que la cirugía esté contraindicada ${ }^{3}$.

El metanálisis de Andersen 2009 mostró la efectividad de la profilaxis con antibióticos en la prevención de las complicaciones pos-operatorias en pacientes con apendicectomía. Lo anterior se debería considerar para el uso habitual en la apendicectomía de urgencia. No todos los estudios informaron ni utilizaron antibióticos profilácticos. Sólo dos estudios informaron el uso de antibióticos profilácticos. No utilizar antibióticos profilácticos en el grupo de apendicectomía causaría una tasa mayor de complicación (principalmente infecciones de la herida) en el grupo de apendicectomía. Ninguno de los ensayos diferenció las complicaciones entre la apendicectomía abierta y laparoscópica ${ }^{2}$. 
Un metanálisis mostró una diferencia en las complicaciones entre la cirugía laparoscópica y abierta para la apendicitis aguda. Se informaron más infecciones de la herida en la cirugía abierta y más abscesos intra-abdominales en la apendicectomía laparoscópica. También la experiencia del cirujano tuvo una función fundamental.

Manejo quirúrgico de apendicitis complicada sin colocación de drenajes ${ }^{4}$ realizada por Rojas, L., et al, los resultados más sobresalientes fueron que de los 97 manejados sin drenaje, 8 presentaron complicaciones (8.2\%): fiebre en tres, seroma en uno, infección superficial del sitio quirúrgico en dos e infección profunda del sitio quirúrgico (formación de colecciones intra-abdominales) en dos. Todos los pacientes respondieron a manejo terapéutico, sin reintervención. Conclusión: El porcentaje de complicación fue de $8.2 \%$, menor a lo reportado en la literatura revisada

En el trabajo de Saade, R. et al, Tratamiento laparoscópico de la apendicitis aguda en la Cátedra de Clínica y Terapéutica Quirúrgica C, de la Escuela de Medicina Luís Razetti, Servicio de Cirugía III del Hospital Universitario de Caracas concluyeron que la tasa de complicaciones fue de 9,68 \%, siendo la más frecuente la infección de la pared abdominal (6,99 \%), el absceso intraabdominal $(1,62 \%)$ y la celulitis de la pared abdominal (1,02 \%). El porcentaje de reconversión fue de 3,76 \%. La mortalidad fue de $0 \%$. La apendicectomía laparoscópica constituye un método seguro y efectivo para el tratamiento de la apendicitis aguda ${ }^{5}$.

En la investigación realizada por Alarcón, E- Bustamante, K., Correlación entre el diagnóstico clínico y de laboratorio de apendicitis aguda frente a los hallazgo anatomopatológicos en pacientes hospitalizados en el área de cirugía del hospital IEES de Manta Mayo 2012- febrero 2013, las conclusiones fueron existen más casos de apendicitis aguda en el sexo masculino, en un rango de edades de 17 a 29 años. Se observó en este estudio que existieron caos de apéndice normal 
en un porcentaje bajo, debido a que se hace un buen examen físico y datos de laboratorio que ayudan a corroborar el diagnóstico ${ }^{6}$.

Las conclusiones de este trabajo fueron que los/as pacientes para acuden a solicitar atención médica en forma directa sin cumplir con el sistema de referencia y contrarreferencia, ingresaron con diagnóstico presuntivo de apendicitis aguda el 45, 69\% de mujeres y el 54,31\% de hombres. Los grupos más afectados fueron menores de 19 años y de 20 a 29 años. El 66\% buscó atención médica antes de las 24 horas de la presentación de síntomas o signos, el 13\% entre 25 a 48 horas y el $21 \%$ después de las 49 horas. El 16\% tuvo alguna co-morbilidad, de los cuales el 11\% presentó diabetes en todos los grupos de edad. Los signos presentes en la valoración fueron Blumberg 28,45\%; Mc Burney 25,86\%; Murphy 8,62; Blumberg -Mc Burney 15,52\%; Mc Burney - Murphy 13,79\%; Blumberg -Mc Burney- Murphy 5,17\% y Blumberg - Murphy 2,59\%. Las alteraciones hematológicas fueron Leucocitosis $\geq 10000 \mathrm{~mm} 366,37 \%$; Glicemia $\geq 120 \mathrm{mgl} 44$ \%; Creatinina sérica $\geq 1.1 \quad 3,45$, estas alteraciones se presentaron en forma combinada. Los antibióticos más utilizados fueron Ceftriaxona 36,21\%, Amplicilina 20,69\% Amplicilina sulbactam 8,62 \% en menores porcentajes otros antibióticos y no recibieron medicación profiláctica 26,72\%. El 96\% de pacientes recibió anestesia general y el 4\% raquídea. Las complicaciones intra-operatoria fueron contaminación contenido apendicular en el 93\% y con el 3, 45 \% contaminación por coprolito y hemorragia controlada, respectivamente. Las complicaciones post operatorias fueron $81 \%$ infección de la herida, abscesos intra-abdominales $11 \%$ y hematoma de pared el $8 \%$. El $75 \%$ de los pacientes fue operado entre 1 a 4 horas posteriores al ingreso. De 5 a 8 horas el 11\% y más de 12 h en $13 \%$.El diagnóstico definitivo fue Apendicitis aguda con peritonitis generalizada 48\%, Apendicitis aguda con absceso peritoneal $11 \%$ y $40 \%$ apendicitis aguda. 
El presente estudio pretende establecer la incidencia de apendicitis aguda y proponer un protocolo para el manejo de esta patología para el área de Emergencia del Hospital Guayaquil- Dr. Abel Gilbert Pontón. (HAGP)

\section{Materiales y métodos.}

\section{Localización}

Se trabajó en el servicio de Emergencia del HAGP-" ubicado en la calle 29 y Galápagos, (Suburbio oeste) de la Parroquia Febres Cordero de Guayaquil-Ecuador.

\section{Caracterización de la zona de trabajo}

El hospital fue planificado en la 5ta Presidencia del Dr. José María Velasco Ibarra encargando a la Constructora CONASA, la edificación y equipamiento, con la modalidad de "llave en mano"

La Dictadura Militar de Rodríguez Lara recibe el hospital concluido, siendo Ministro de Salud el Coronel de EM Dr. Raúl Maldonado Mejía + y nombrado Director de ese centro asistencial el Dr. Eduardo Iglesias Espinel se inauguró el 7 de Octubre de 1973, con el nombre de Hospital Guayaquil, hasta ese momento conocido como Hospital del Suburbio. El primer año funcionó la Consulta Externa y un área de atención de Emergencias y Fisiatría.

En ese período se realizan los nombramientos de las Jefaturas de los Servicios, ganando por concurso la Jefatura de Fisiatría Dr. Fidel Endara Cedeño, de Emergencia y Cirugía Dr. Teófilo Lama Pico, de Pediatría Dr. Luís Sarracín Dávila, de Medicina Interna Dr., Xavier Manrique Trujillo +, de Obstetricia Dr. Octavio Ortiz Peralta. En la Jefatura de Enfermería el nombramiento 
recayó sobre la Enfermera especializada en Cuidados Intensivo Lupe Quiroz Villarreal y en las jefaturas de los servicios Enfermería de Emergencia y Cirugía señora Norma Baquerizo de Rosarión, Pediatría Lic. Gladys Jarrín Córdova, Gineco - obstetricia Lic. Lourdes Muñoz Ortiz, de Consulta Externa Enf. Luz Chamba y en cuidado directo 25 enfermeras egresadas de la Escuela de Enfermería de la Universidad de Guayaquil, que cumplían el año de Salud Rural y 40 auxiliares de enfermería certificadas.

Desde 1973 hasta abril de 1975, se inició la atención en emergencia, hospitalización de Pediatría, Neonatología, Ginecología, Medicina Interna y parcialmente Sala de operaciones y hospitalización Cirugía, funcionando con su capacidad instalada en 1978.

\section{Análisis de la información}

Este trabajo se realizó con la revisión de historias clínicas de pacientes atendidos en la Emergencia del Hospital Guayaquil- Dr. Abel Gilbert Pontón, dependiente del Ministerio de Salud Pública, año 2013.

La base de datos se elaboró en Excel y los resultados se obtuvieron utilizando el paquete estadístico OPS - Epi Info 3.5.1- 2008. Se trabajará con una confiabilidad del 95\% - p<0.05.

Se realizó la descripción de frecuencias absolutas y relativas de cada variable En las variables cualitativas se estiman los estadísticos de interés (media \pm , promedio, desviación estándar) Las asociaciones entre variables categóricas fueron evaluadas mediante la prueba de Chi cuadrado de Pearson ( $\mathrm{n}$ x k). 


\section{Resultados.}

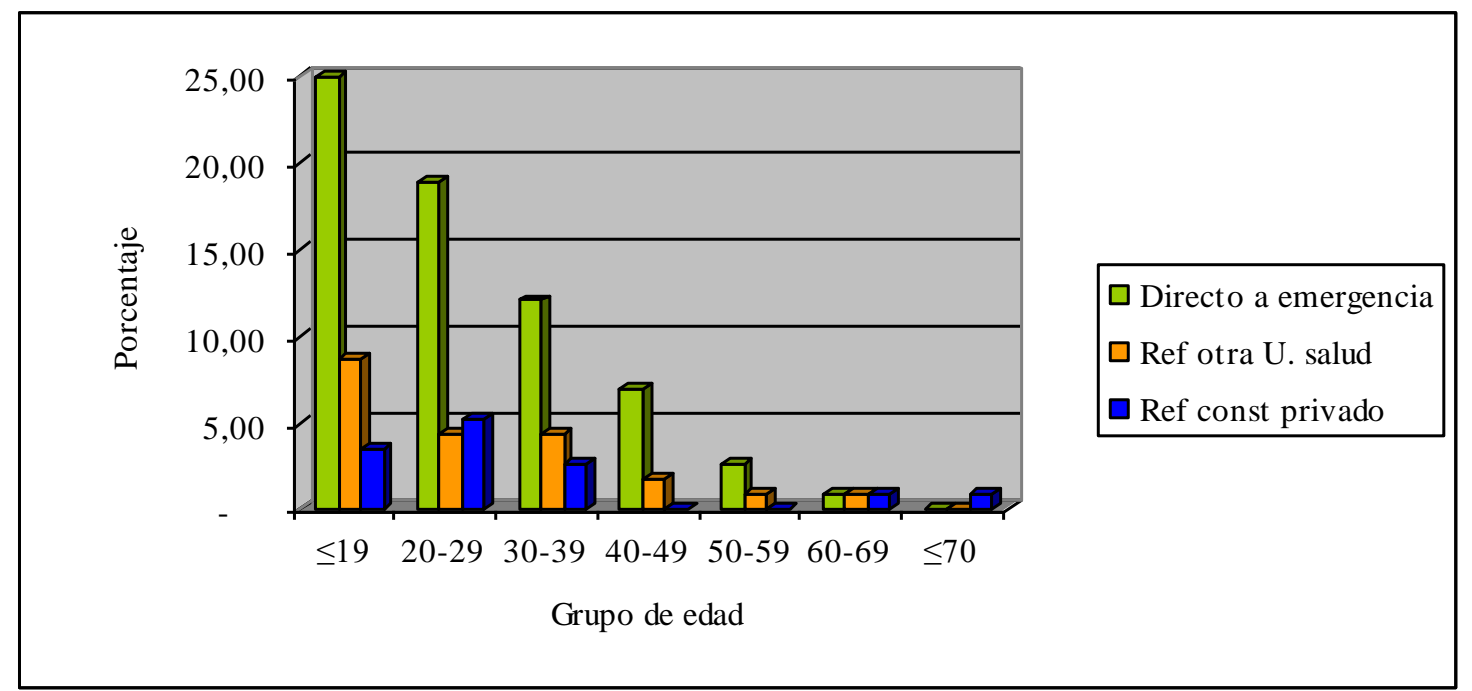

\section{Gráfico $N^{o}$ 1.- Forma de ingreso a Emergencia de los pacientes de apendicitis}

La mayoría de pacientes acuden directamente al Hospital, el 66,38\%, 20,69 \% referidos por unidades de salud y $12,93 \%$ atendidos en un consultorio privado.

Lo que evidencia que la mayoría de los usuarios buscan atención hospitalaria sin utilizar los servicios de atención primaria, debido a que la población no está educada en la utilización del sistema de referencia y contrarreferencia, lo que hace que el servicio de emergencia del hospital siempre esté sobresaturado, y que muchas ocasiones se retrase la tención 


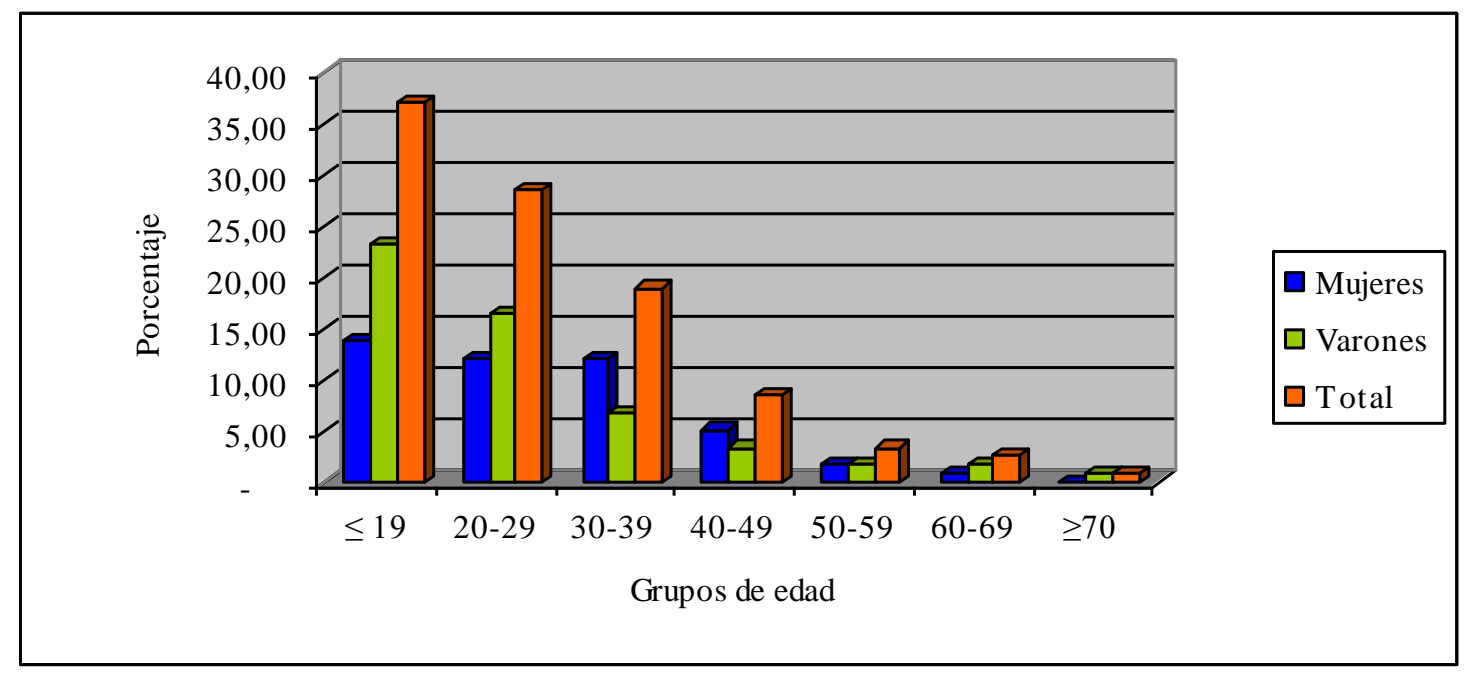

\section{Gráfico $N^{o}$ 2.- Género y grupos de edad de los pacientes con diagnóstico de apendicitis que}

\section{ingresaron a Emergencia}

Los/as pacientes que ingresaron a Emergencia con diagnóstico presuntivo de apendicitis aguda fueron de género femenino el 45, $69 \%$ y masculino el 54,31\%.

En el grupo de mujeres la edad mínima fue 15 años y la máxima 65 años con un promedio de 28,38 años DE 11.68. En los varones se presentó la patología entre los 14 a 73 años con un promedio de 27,22 años DE 12,52. Los grupos de edad más afectados por la patología fueron menores de 19 años y de 20 a 29 años, en el grupo de mujeres representó el 26\%, en varones el $40 \%$ y en el grupo total el $65 \%$. No hay diferencias significativas entre edades promedios.

Artajona Rosino, $\mathrm{A}^{4}$ en el trabajo Apendicitis aguda refiere que existe un pico de máxima incidencia fue entre los 10 y los 20 años de edad y que constituye la causa más frecuente de abdomen agudo en el adulto joven, situación que se evidencia en este trabajo. 


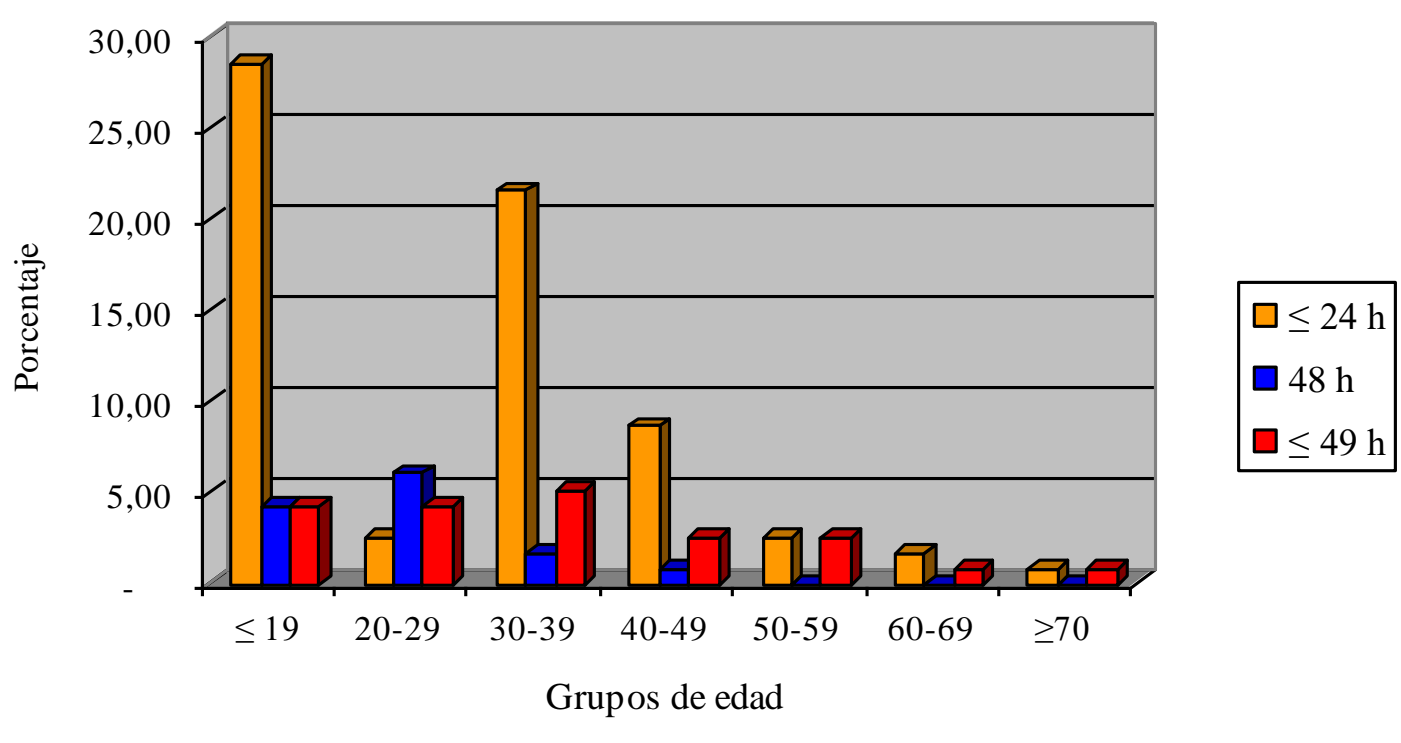

Gráfico $N^{o}$ 3. - Presencia de signos y síntomas de apendicitis aguda por grupos de edad y tiempo de espera para buscar atención médica

Los/as pacientes de este estudio el 66\% refiere que ha buscado atención médica antes de las 24 horas de la presentación de síntomas o signos, el 13\% entre 25 a 48 horas y el $21 \%$ después de las 49 horas. Al cruzar esta información con las complicaciones post quirúrgicas el 100\% presenta éstas, lo que posiblemente se deba a que consideran que los signos y síntomas se presentaron cuando fueron muy severos y es ahí cuando buscan la atención médica.

En la investigación de Cabrejos Pita, J. Factores causantes y consecuencias de la demora en el tratamiento quirúrgico de pacientes con apendicitis aguda en el Hospital de Apoyo de Chachapoyas - Perú entre 1995 y 2000, se atribuyó la responsabilidad de la demora al paciente cuando éste acudió o fue conducido al Hospital para el tratamiento definitivo 72 o más horas después del inicio de los síntomas por decisión del propio paciente o de los familiares de éste. 
En los dos estudios hay diferencia en el tiempo en que acuden los pacientes a recibir atención médica.

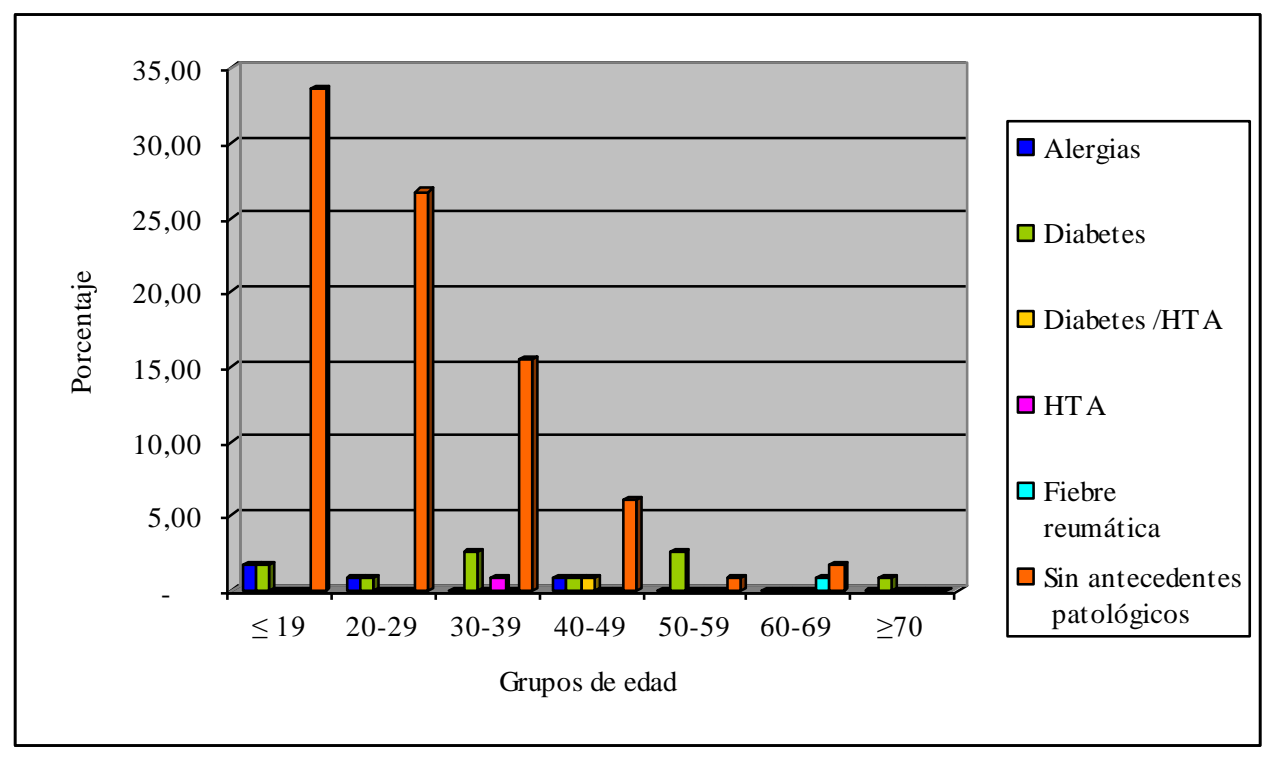

Gráfico $N^{o}$ 4.- Co morbilidad de los pacientes de apendicitis aguda ingresados a Emergencia

El 16\% tuvo alguna co-morbilidad, de los cuales el $11 \%$ presentó diabetes en todos los grupos de edad, alergias HTA, fiebre reumática en porcentajes menores que representaron en forma agrupada el 5\% y sin antecedentes patológicos el $84 \%$.

En el estudio de Tsai, SH, et al, ${ }^{33}$ apendicitis aguda complicada en pacientes diabéticos refieren que en 1184 pacientes con diagnóstico de egreso primario de apendicitis aguda, se halló 71 pacientes con antecedentes de diabetes. Los datos demográficos demostraron que los pacientes diabéticos eran adultos mayores de 60 años. 
Llama la atención que en los pacientes del HAGP la diabetes se presentó mayoritariamente en población joven, $8 \%$ entre menores de 19 años a 49 años y sólo el 3\% en población mayor de 50 años, a excepción del grupo de 60-69 años con 0 casos, contrario al estudios de Tsai y a lo que reporta la literatura que la Diabetes afecta mayoritariamente a población adulta mayor.

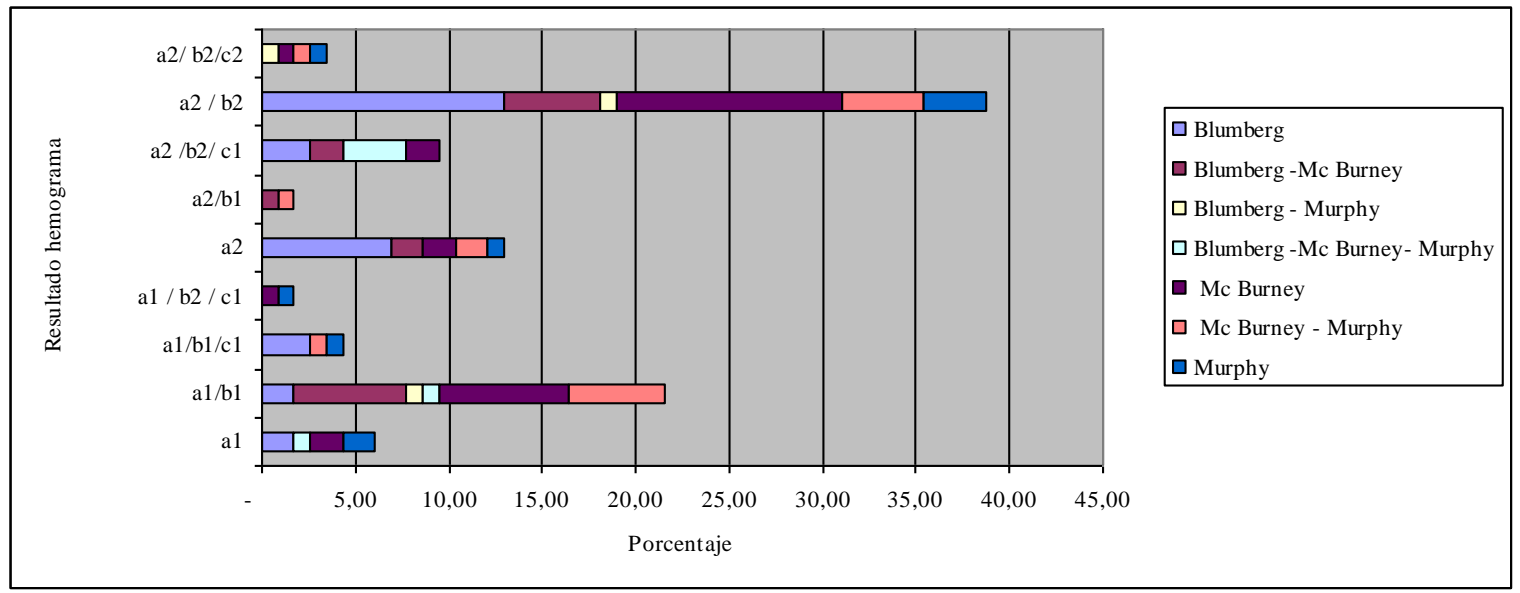

Clave de alteraciones hematológicas
a1. Leucocitosis $\leq 9900 \mathrm{~mm} 3$
b1 Glicemia $>120 \mathrm{mgl}$
c1 Creatinina sérica $<1.1$
a2. Leucocitosis $\leq 10000 \mathrm{~mm} 3$
b2 Glicemia $\leq 120 \mathrm{mgl}$
c2 Creatinina sérica $\geq 1.1$

\section{Gráfico $N^{\circ}$ 5.- Signos y alteraciones hematológicas en los pacientes de apendicitis aguda que}

\section{ingresaron a Emergencia}

Los signos presentes en la valoración fueron signo de Blumberg 28,45\%; Mc Burney 25,86\%; Murphy 8,62\%; Blumberg -Mc Burney 15,52\%; Mc Burney - Murphy 13,79\%; Blumberg Mc Burney- Murphy 5,17\% y Blumberg - Murphy 2,59\%. Las alteraciones hematológicas fueron Leucocitosis $\geq 10000 \mathrm{~mm} 366,37 \%$; Glicemia $\geq 120 \mathrm{mgl}$. $44 \%$; Creatinina sérica $\geq 1.1 \quad 3,45$, estas alteraciones se presentaron en forma combinada. 
Rivera Díaz, EM., en la investigación Evaluación comparativa del tratamiento quirúrgico de la apendicitis aguda: Apendicectomía abierta versus apendicectomía laparoscópica en el Hospital Nacional Carlos A. Seguín E. Essalud - Año 2,000, el 49.5\% de casos, grupo total, tienen leucocitos mayor de 10,000/mm3. El 81\% de casos, grupo total, tienen glicemia menor de $124 \mathrm{mg} / \%$ y el $19 \%$ presenta glicemia alta y el $88 \%$ de casos, tienen creatinina sérica menor de 1.1 . El $12 \%$ de casos, grupo total, tienen Creatinina Sérica mayor de 1.2. En estos dos trabajos difiere los porcentajes de pacientes con leucocitos $\geq 10000 / \mathrm{mm} 3$ y glicemia elevada.

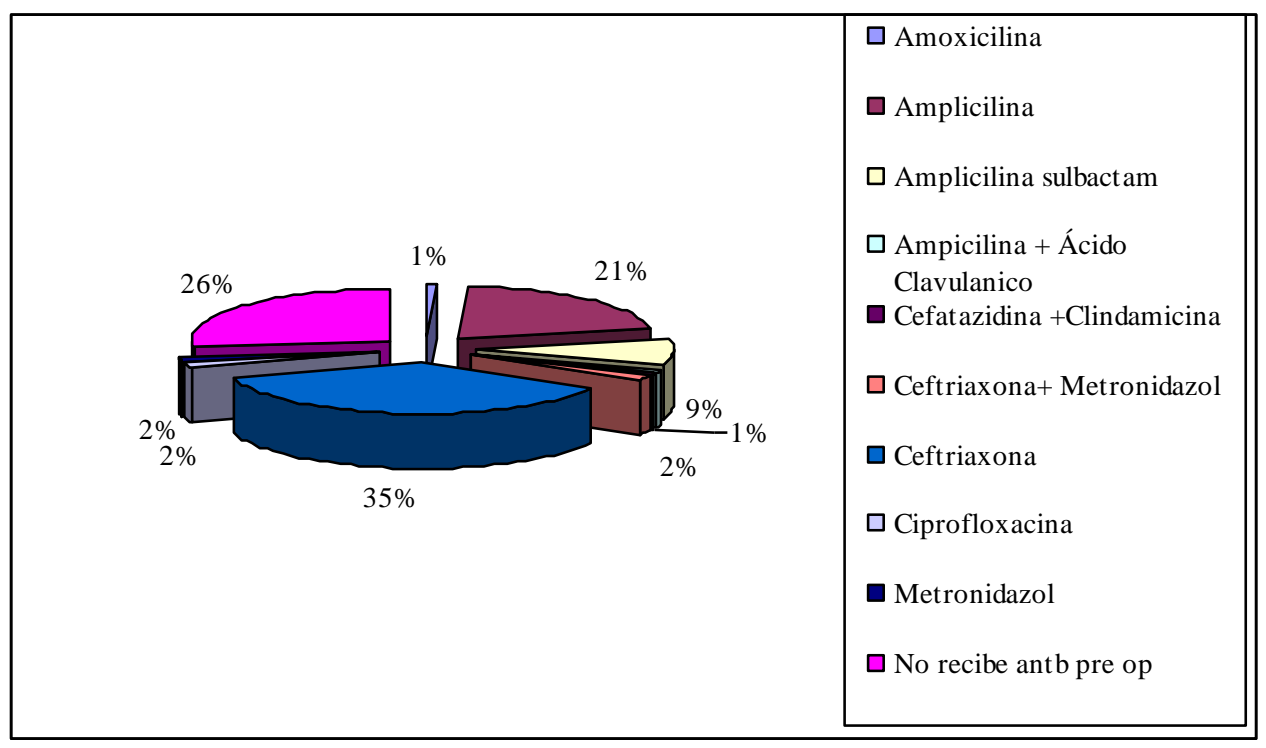

\section{Gráfico $N^{0}$ 6.- Profilaxis antibiótica a pacientes de apendicites aguda}

Los antibióticos más utilizados fueron Ceftriaxona 36,21\%, Amplicilina 20,69\% Amplicilina sulbactam 8,62 \% en menores porcentajes Amoxicilina, Ácido Clavulanico Cefatazidina, Clindamicina, Metronidazol, Ciprofloxacina y no reciben medicación profiláctica 26,72\%. 
La adecuada selección y el uso de antibióticos son primordiales en el manejo de apendicitis; sin embargo, el uso de éstos para el manejo de los pacientes muchas veces se ve truncado, debido a la existencia y disponibilidad en el momento de diagnóstico del cuadro; sin embargo siempre se buscó la cobertura de la flora bacteriana colónica más frecuente.

Rivera Díaz, EM., en la investigación Evaluación comparativa del tratamiento quirúrgico de la apendicitis aguda: Apendicectomía abierta versus apendicectomía laparoscópica en el Hospital Nacional Carlos A. Seguín E. Essalud - Año 2,000, el 51\% de casos, grupo total, recibieron antibióticos en el preoperatorio. Igual. Los Antibióticos usados: Amikacina 1 gr. c/24 h en los no complicados y Amikacina (1 gr. c/24 h) + Clindamicina (600 mg c/8 hrs.) en los complicados.

En el HAGP el 73\% de pacientes recibe medicación antibiótica porcentaje mayor que el dado en el estudio con el que se comparan los datos.

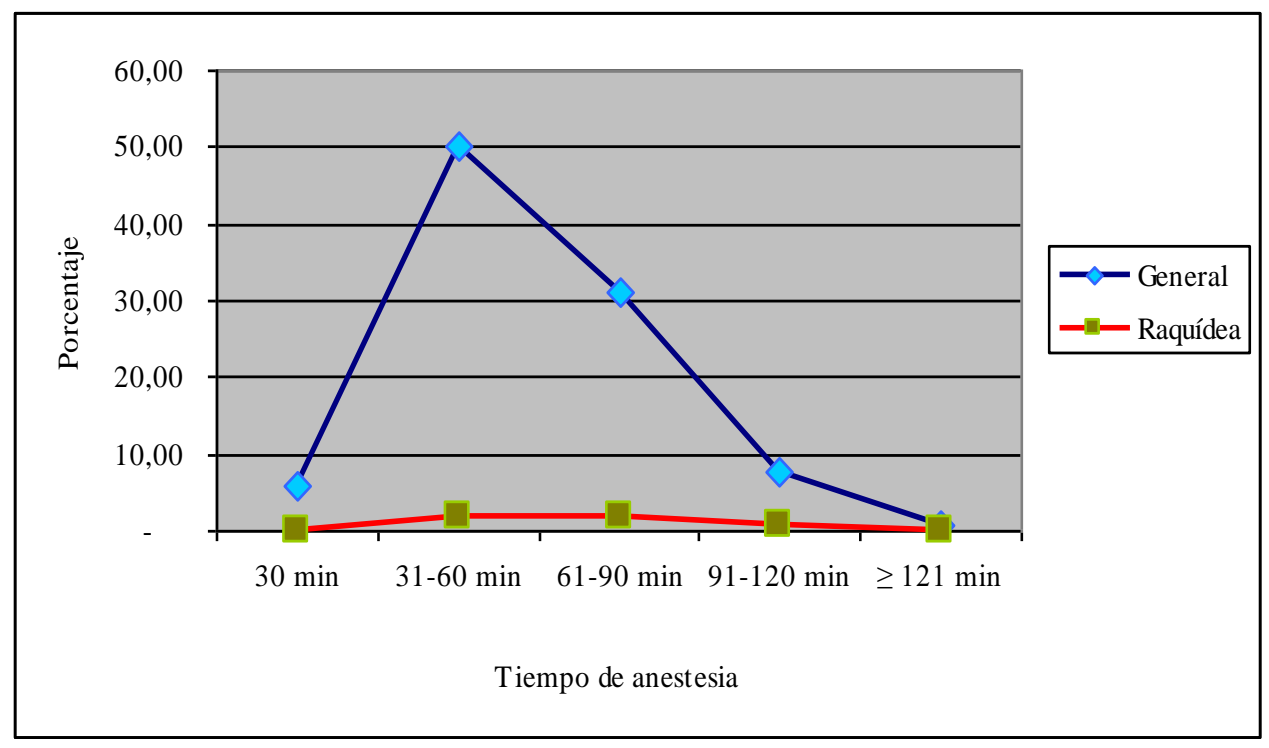

Gráfico $N^{o}$ 7.- Tipo y tiempo de duración de la anestesia en la cirugía de apendicectomía 
El tipo de anestesia utilizada la elige el anestesiólogo previo a una valoración del grado de riesgo anestésico. En esta serie el $96 \%$ de pacientes recibió anestesia general siendo los tiempos de cirugía entre $31-60 \min .52 \%$ y $61-90 \min .33 \%$ y con porcentajes menores los tiempos menos de 30 min, 91 a 120 min. y más de 120 min. La anestesia raquídea sólo se utilizó en el 4\% de los pacientes.

En la investigación de Farfán Espinosa, O. 2002 Apendicitis Aguda en el Hospital Dos de Mayo, la anestesia más usada fue la de tipo regional, 62,68\% raquídea y epidural 4\%. La anestesia general se utilizó en el 33,3\% ${ }^{7}$.

Comparando los dos estudios se ve una diferencia en el uso de la anestesia, la misma que puede estar dada por la diferencia en la experticia de los anestesiólogos o grado de riesgo con que ingresan los pacientes a la cirugía.

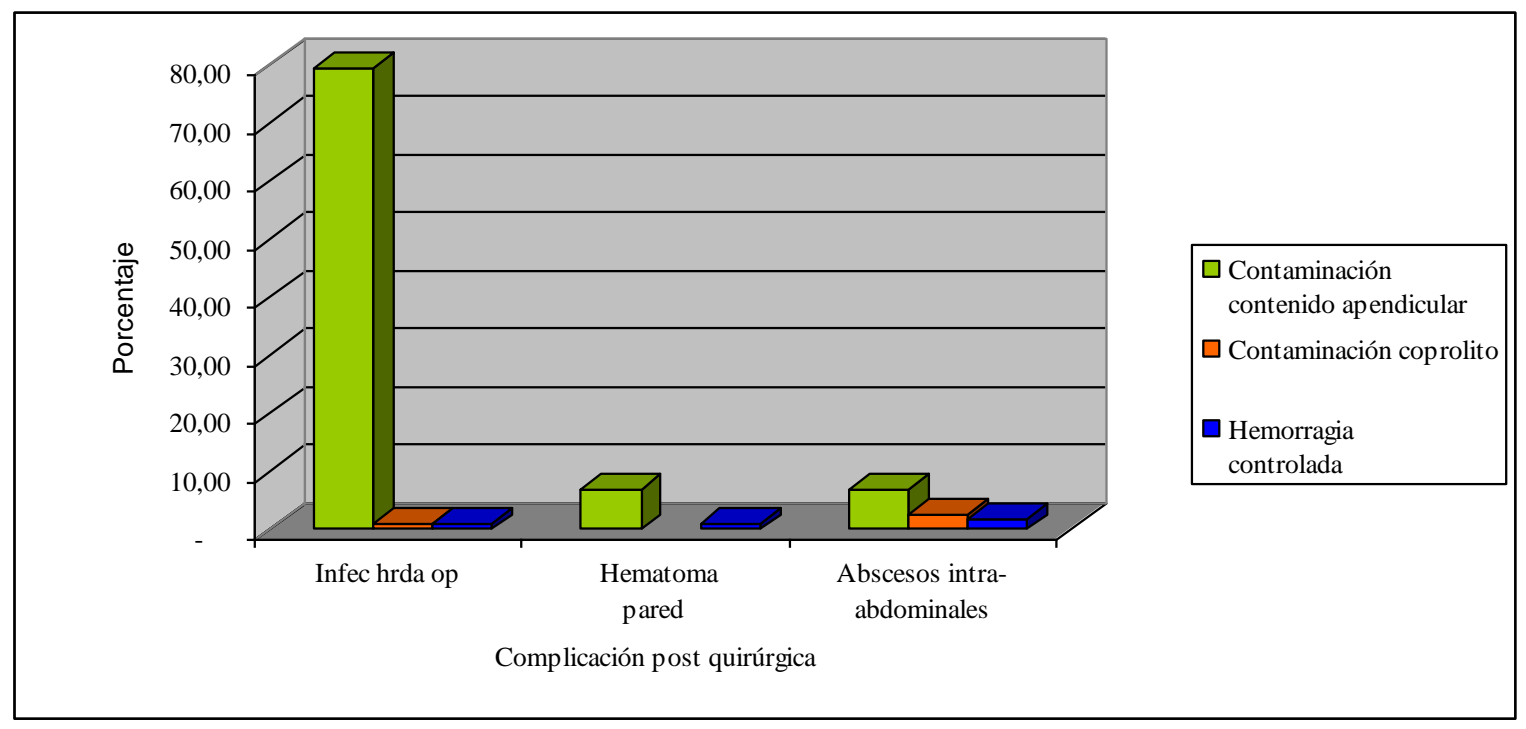

Gráfico $N^{o}$ 8.- Complicaciones intra y post operatorias en apendicetomías de emergencia 
En los pacientes de apendicitis aguda de emergencia del HAGP las complicaciones intraoperatoria más frecuente fueron: contaminación contenido apendicular 93\% y 3, $45 \%$ la contaminación por coprolito y hemorragia controlada, respectivamente. Con relación a las complicaciones post operatorias el $81 \%$ presentó infección de la herida, abscesos intra-abdominales el $11 \%$ y hematoma de pared el $8 \%$.

En la investigación de Rivera Díaz, EM., Evaluación comparativa del tratamiento quirúrgico de la apendicitis aguda: Apendicectomía abierta versus apendicectomía laparoscópica en el Hospital Nacional Carlos A. Seguín E. Essalud - Año 2,000, los accidentes intraoperatoria corresponde a contaminación contenido apendicular o coprolito y hemorragia controlada. La mayor frecuencia corresponde a la Apendicectomía laparoscópica con 10\%, diferencia con significación estadística.

En la investigación de Farfán Espinosa, O. 2002 Apendicitis Aguda en el Hospital Dos de Mayo las complicaciones post operatorias fueron infección de la herida operatoria en el 15,21\%, y entre los factores que influyeron el alto número de pacientes con apendicitis aguda perforada. Otra complicación fueron los abscesos intra-abdominales o pélvicos en el $0,96 \%{ }^{7}$.

Comparando los trabajos de Rivera Díaz y el del HAGP las complicaciones intra-operatorias son similares en los dos estudios y en relación a las complicaciones post operatorias son mayores en los pacientes del HAGP que los tratados con la misma patología, en el Hospital Dos de Mayo. 


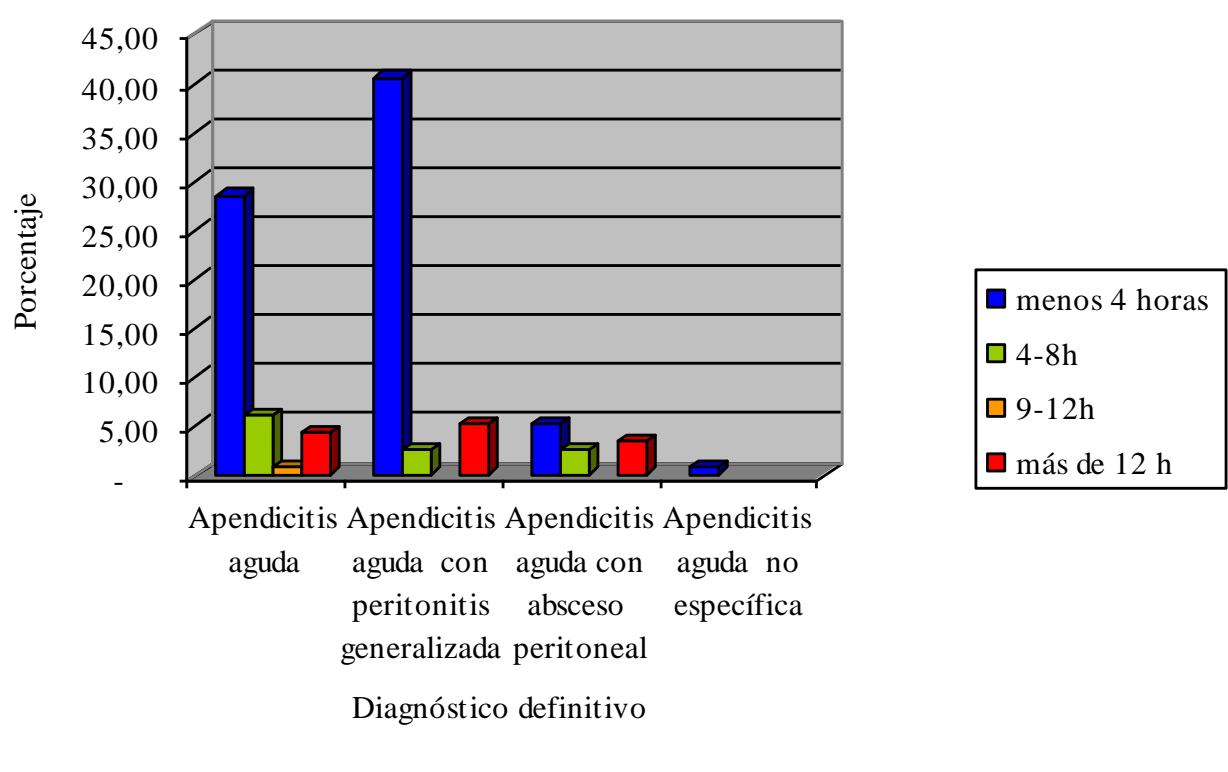

Gráfico $N^{o}$ 9.- Diagnóstico definitivo y tiempo de espera de la cirugía en pacientes de apendicitis aguda

El tiempo de espera para la cirugía, (tiempo de demora entre el ingreso y la cirugía) es un factor importante que sumadas al tiempo que demora el paciente en acudir al centro asistencial para buscar asistencia médica contribuye al incremento del porcentaje de complicaciones intra y post quirúrgicas.

El 75\% de los pacientes fue operado entre 1 a 4 horas posteriores al ingreso, pero a pesar de ello el $41 \%$ tuvo diagnóstico apendicitis aguda con peritonitis generalizada, y apendicitis aguda con absceso peritoneal el 5\%; los pacientes que fueron operados en un tiempo mayor a las 12 horas de ingreso, el $13 \%$ tuvo como diagnóstico definitivo apendicitis aguda con peritonitis generalizada $5 \%$ y apendicitis aguda con absceso peritoneal el 4\%. El 40\% de los pacientes de esta serie tuvieron diagnóstico de apendicitis aguda. 
En estudios realizados en Hospitales de Lima -Perú el tiempo de espera promedio fue de 7, 45 horas en el Hospital Hipólito Unanue, 7,55 horas Hospital Nacional Arzobispo Loyola y en el Hospital Dos de Mayo 11 horas, en este hospital el 49\% tuvo diagnóstico de apendicitis aguda complicada ${ }^{7}$.

\section{Discusión.}

La apendicitis aguda es una patología que se presenta en ambos sexos con predominio del sexo masculino, con una relación de 1,3/1 hombres /mujeres con una incidencia similar que la reportada en la bibliografía consultada. Los gripos de dad más afectados fueron los menores de 19 años y de 20 a 29 años. El grupo de 60 años que representó el 3,5\% del total de la población en estudio sin ser un grupo numeroso es un grupo en el que debe ponerse atención por los factores de riesgo propios de la edad. Sin embargo en este trabajo se evidencia la presencia de diabetes en todos los grupos de edad inclusive en los menores de 19 años alcanzando el 10, $46 \%$ de la co-morbilidad presente y con porcentajes muy bajos HTA, alergias, fiebre reumática y sin antecedentes patológicos el $85 \%$.

El retraso en el diagnóstico puede desencadenar complicaciones que llevarán al paciente a un deterioro físico, aumento en la estancia hospitalaria, elevación de costos para los servicios de salud, retardo en la reincorporación a sus actividades cotidianas, e incluso pueden llevarlo a la muerte ${ }^{8,9,10}$.

Al no diagnosticar a tiempo un cuadro de apendicitis aguda, éste puede complicarse y desarrollar rápidamente perforación, absceso peri- apendicular,17 entre otros. Para hacer frente a las complicaciones se ha buscado aumentar la exactitud diagnóstica, esto se ha logrado apoyando el diagnóstico en estudios complementarios, tales como ultrasonido demostrando sensibilidad y especificidad de 80 a $89 \%$. 
La anestesia más utilizada fue la general en el $96 \%$ de los casos y el $4 \%$ de raquídea, la misma que es decisión del anestesiólogo, previa la valoración del riesgo anestésico. En este trabajo no se reporta hallazgos de apéndice sano, se reportó contaminación de contenido apendicular en el 93\%, contaminación con coprolito y hemorragia controlada en el 3,45\% respectivamente.

A lo largo de los años, la clasificación de la apendicitis aguda ha sido tema de debate. Para valorar la gravedad de la apendicitis existen diferentes clasificaciones con bases clínicas e histológicas, sin embargo, esta clasificación no tiene un sustento bibliográfico, por lo que algunos autores utilizan otras: apendicitis aguda complicada o no complicada, apendicitis aguda perforada o no perforada. Esto demuestra que no existe un consenso unificado para su clasificación.15 Para este trabajo se utilizó la clasificación del CIE 10 K35 Apendicitis aguda 40\% K35.1 Apendicitis aguda con peritonitis generalizada $11 \%, \mathrm{~K} 37$ Apendicitos aguda no específica $1 \%$. En el período en estudio no se reporta mortalidad en pacientes operados de apendicitos aguda.

\section{Conclusiones.}

Los/as pacientes para acuden a solicitar atención médica lo hacen en forma directa al Hospital, el 66,38\%, 20,69 \% referidos y cumplen con el sistema de referencia y el 12,93\% atendidos previamente en una consultorio privado.

Los/as pacientes que ingresaron a Emergencia con diagnóstico presuntivo de apendicitis aguda fueron de género femenino el $45,69 \%$ y masculino el 54,31\% del masculino. La patología se presentó entre los 14 a 73 años No hay diferencias significativas entre edades promedios. Los grupos de edad más afectados por la patología fueron menores de 19 años y de 20 a 29 años, en las mujeres representó el $26 \%$, en varones el $40 \%$ 
Los/as pacientes de este estudio el $66 \%$ buscó atención médica antes de las 24 horas de la presentación de síntomas o signos, el 13\% entre 25 a 48 horas y el $21 \%$ después de las 49 horas.

El 16\% tuvo alguna co-morbilidad, de los cuales el 11\% presentó diabetes en todos los grupos de edad, alergias HTA, fiebre reumática en porcentajes menores que representaron en forma agrupada el 5\% y sin antecedentes patológicos el $84 \%$.

Los signos presentes en la valoración fueron signo de Blumberg 28,45\%; Mc Burney 25,86\%; Murphy 8,62; Blumberg -Mc Burney 15,52\%; Mc Burney - Murphy 13,79\%; Blumberg Mc Burney- Murphy 5,17\% y Blumberg - Murphy 2,59\%. Las alteraciones hematológicas fueron Leucocitosis $\leq 10000 \mathrm{~mm} 366,37 \%$; Glicemia $\leq 120 \mathrm{mgl} 44 \%$; Creatinina sérica $\geq 1.1 \quad 3,45$, estas alteraciones se presentaron en forma combinada.

Los antibióticos más utilizados fueron Ceftriaxona 36,21\%, Amplicilina 20,69\% Amplicilina sulbactam 8,62 \% en menores porcentajes Amoxicilina, Ácido Clavulanico Cefatazidina, Clindamicina, Metronidazol, Ciprofloxacina y no reciben medicación profiláctica 26,72\%.

El 96\% de pacientes recibió anestesia general siendo los tiempos de cirugía entre 31 -60 min. $52 \%$ y $61-90$ min. $33 \%$ y con porcentajes menores los tiempos menos de 30 min. , 91 a 120 min. y más de 120 min. La anestesia raquídea sólo se utilizó en el 4\% de los pacientes.

La complicación intra-operatoria más frecuente fue la contaminación contenido apendicular en el 93\% y con el 3, $45 \%$ la contaminación por coprolito y hemorragia controlada, respectivamente. Con relación a las complicaciones post operatorias el 81\% presentó infección de la herida, abscesos intra-abdominales el 11\% y hematoma de pared el $8 \%$. 
El $75 \%$ de los pacientes fue operado entre 1 a 4 horas posteriores al ingreso, en este subgrupo el $41 \%$ tuvo diagnóstico apendicitis aguda con peritonitis generalizada, y apendicitis aguda con absceso peritoneal; el 5\%; los pacientes que fueron operados en un tiempo mayor a las 12 horas de ingreso, el $13 \%$ tuvo como diagnóstico definitivo apendicitis aguda con peritonitis generalizada $5 \%$ y apendicitis aguda con absceso peritoneal el $4 \%$. El $40 \%$ de los pacientes de esta serie tuvieron diagnóstico de apendicitis aguda.

\section{Bibliografía.}

1. Arias E J. 2006 Apendicitis. Experiencia del Hospital Ramón González Valencia. Rev. Col Cir.; 9 (4) pp. 201-206.

2. Andersen BR, Kallehave FL, Andersen HK. Antibióticos versus placebo para la prevención de la infección pos-operatoria después de una apendicectomía (Revisión Cochrane traducida). En:La Biblioteca Cochrane Plus, 2008 Número 4. Oxford: Update Software Ltd. Disponible en: http://www.update-software.com. (Traducida de The Cochrane Library, 2008 Issue 3. Chichester, UK: John Wiley \& Sons, Ltd.).

3. Wilms I, de How D, de Visser D, Janzing H. Apendicectomía versus tratamiento con antibióticos para la apendicitis aguda. Cochrane Database of Systematic Reviews 2011 Issue 11. Art. No.: CD008359.

4. Rojas, L., et al. Manejo quirúrgico de apendicitis complicada sin colocación de drenajes. Sonora México. Hospital General del Estado Dr. Ernesto Ramos Tours. Revista Cirugía General Vol. 34 № 3 julio/ sep 2012.

5. Saade, R. et al 2007 Tratamiento laparoscópico de la apendicitis aguda en la Cátedra de Clínica y Terapéutica Quirúrgica C, de la Escuela de Medicina, Luís Razetti, Servicio de Cirugía III del Hospital Universitario de Caracas.

6. Alarcón, E- Bustamante, K. Correlación entre el diagnóstico clínico y de laboratorio de apendicitis aguda frente a los hallazgo anatomopatológicos en pacientes hospitalizados en el área de cirugía del hospital IEES de Manta Mayo 2012- febrero 2013. Manta - Ecuador. Universidad Laica Eloy Alfaro. Escuela de Medicina Tesis de grado.

7. Farfán Espinosa, O. 2002 Apendicitis Aguda en el Hospital Dos de Mayo. Enero 2000- julio 2001 del Lima -Perú Universidad Nacional Mayor de San Marcos- Facultad de Medicina, Escuela de Post Grado. Trabajo de Investigación para optar por el Título de Especialista en Cirugía General.

8. Vissers RJ, Lennarz WB. Pitfalls in appendicitis. Emerg Med Clin North Am 2010; 28: pp. 103-118.

9. Pérez, MA. Ramírez, HI. Suárez, N. Álvarez Y. Paniagua, E. Morbimortalidad por apendicitis complicada en el Hospital Padre Billini. Rev. Med Dom. 2003; 68 pp. 133-136

10. Temple CL, Huchcroft SA, Temple WJ. The natural history of appendicitis in adults. A prospective study. Ann Surg 1995; 221 pp. 278-281. 